\title{
Population, urbanization and economic scenarios over the Belt and Road region under the Shared Socioeconomic Pathways
}

\author{
JING Cheng ${ }^{1,4}$, TAO Hui ${ }^{1}$, "JIANG Tong ${ }^{2}$, WANG Yanjun ${ }^{2}$, ZHAI Jianqing ${ }^{2,3}$, \\ CAO Lige ${ }^{3}$, SU Buda ${ }^{1,2,3}$
}

1. State Key Laboratory of Desert and Oasis Ecology, Xinjiang Institute of Ecology and Geography, CAS, Urumqi 830011, China;

2. Collaborative Innovation Center on Forecast and Evaluation of Meteorological Disasters/Institute for Disaster Risk Management/School of Geographical Science, Nanjing University of Information Science \& Technology, Nanjing Jiangsu 210044, China;

3. National Climate Center, China Meteorological Administration, Beijing 100081, China;

4. University of Chinese Academy of Sciences, Beijing 100049, China

\begin{abstract}
The countries throughout the Belt and Road region account for more than $60 \%$ of the world's population and half of the global economy. Future changes in this area will have significant influences on the global economic growth, industrial structure and resource allocation. In this study, the proportion of the urban population to the total population and the gross domestic product were used to represent the levels of urbanization and economic development, respectively. The population, urbanization and economic levels of the Belt and Road countries for 2020-2050 were projected under the framework of the IPCC's shared socioeconomic pathways (SSPs), and the following conclusions are drawn. (1) The population, urbanization and economic levels in the Belt and Road region will likely increase under all five pathways. The population will increase by $2 \%-8 \% / 10$ a during $2020-2050$ and reach 5.0-6.0 billion in 2050. Meanwhile, the urbanization rate will increase by $1.4 \%-7.5 \% / 10 \mathrm{a}$ and reach $49 \%-75 \%$. The GDP will increase by 17\%-34\%/10a and reach 134-243 trillion USD. (2) Large differences will appear under different scenarios. The SSP1 and SSP5 pathways demonstrate relatively high urbanization and economic levels, but the population size is comparatively smaller; SSP3 shows the opposite trend. Meanwhile, the economy develops slowly under SSP4, but it has a relatively high urbanization level, while SSP2 exhibits an intermediate trend. (3) In 2050, the population will increase relative to 2016 in most countries, and population size in the fastest growing country in Central Asia and the Middle East countries will be more than double. Urbanization will develop rapidly in South Asia, West Asia and Central Asia, and will increase by more than $150 \%$ in the fastest growing countries. The
\end{abstract}

Received: 2018-12-20 Accepted: 2019-03-26

Foundation: National Key Research and Development Program of China MOST, No.2017YFA0603701; Chinese-Pakistan cooperative project jointly funded by NSFC and PSF, No.41661144027; The CMA Climate Change Science Fund $(201722,201810)$

Author: Jing Cheng (1992-), PhD Candidate, specialized in climate risk assessment. E-mail: jingc1992@163.com

*Corresponding author: Jiang Tong (1962-), Professor, E-mail: jiangtong@nuist.edu.cn 
economy will grow fastest in South Asia, Southeast Asia and West Asia, and increase by more than 10 times in some counties with rapid economic development.

Keywords: population, urbanization and economic scenarios; Shared Socioeconomic Pathways; 2020-2050; the Belt and Road region

\section{Introduction}

The rising overall concentration of greenhouse gases caused by anthropogenic emissions constitutes the main factor contributing to global warming (IPCC, 2013; Rohde et al., 2013; Hansen et al., 2015). Over the past 200 years, industrialization has led to rapid increases in the global population and global economy coincident with widespread urbanization. The burgeoning population, surges in trade and expansion of cities worldwide have caused increases in the emissions of greenhouse gas and the rate of climate change (Weber et al., 2009; Chen et al., 2012; Trenberth et al., 2015). As the current warming trend continues, the negative impacts of the climate and weather extremes on human beings will become increasingly apparent, and more socioeconomic sectors will be exposed to extreme weather events worldwide (Field et al., 2012; Cai et al., 2014; Rosenzweig et al., 2014; Schewe et al., 2014; Donat et al., 2016).

Long-term climate scenarios, the primary and most essential component of climate projections, provide possible descriptions about the future state of the world (van Vuuren et al., 2012; Jones et al., 2014). Climate scenarios can be combined with climate change projections and climate policy assumptions to produce scenarios integrated with climate mitigation, adaptation and impacts (Moss et al., 2010; O’Neill et al., 2017). Since 1990, the Intergovernmental Panel on Climate Change (IPCC) has considered numerous different emission schemes and successively developed emission scenarios of SA90, IS92, and special reports, such as the Special Report on Emissions Scenarios (SRES), etc. (Leggett, 1992; Nakicenovic et al., 2000; Nakicenovic et al., 2006). Among the products of these scenarios, the Representative Concentration Pathways (RCPs) developed in 2007 incorporate the climate-forcing dimensions of different possible futures and form the basis for the development of a new set of parallel scenario process known as Shared Socioeconomic Pathways (SSPs) (van Vuuren et al., 2011; Taylor et al., 2012; Riahi et al., 2017).

SSPs aim to characterize future societal and environmental changes against a reference case. They represent an important complement to quantitative model projections by describing major socioeconomic, demographic, technological, lifestyle, policy, institutional and other trends (Kriegler et al., 2014; O’Neill et al., 2014; Riahi et al., 2017). Five basic SSPs have been developed to describe the challenges of mitigation and adaptation. SSP 1 denotes a sustainable path in which both mitigation and adaptation face few challenges, and SSP2 assumes a world that follows a historical approach with moderate challenges encountered by both mitigation and adaptation. In contrast to SSP1, SSP3 refers to a fragmented world in which great challenges are faced with regard to mitigation and adaptation. SSP4 represents a path of inequality with low challenges to mitigation but high challenges to adaptation. Finally, SSP5 describes a pathway characterized by conventional development with a strong reliance on fossil fuels low challenges to adaptation (O'Neill et al., 2017). The narratives of these five SSPs have quantified and guided the selection of assumptions for various socio- 
economic projections. In particular, in recent years, these SSPs have been used to assess the impacts of the changing environment on agriculture, energy, land use, geopolitics, water scarcity and other fields (Arnell et al., 2014; Fujimori et al., 2014; Hegre et al., 2016; Popp et al., 2017; Mogollón et al., 2018).

For each SSP, a single population (Kc et al., 2014; Lutz et al., 2014; Kc et al., 2017), GDP (Cuaresma, 2017; Dellink et al., 2017; Leimbach et al., 2017) and urbanization (Jiang et al., 2017a) projection was developed. The future socioeconomic changes in about 195 countries have been achieved. However, the previous projection for China was based on statistics from the World Bank and the United Nations that were somehow biased from the actual situation in China. In addition, the SSPs did not consider the impacts of China's new population policy on labour and urbanization. Therefore, we are committed to the study of China's social and economic projections under SSPs by improving the parameters of the population and economic models based on population and economic census data from China and the newly implemented population policy. Accordingly, databases of the population, urbanization and GDP in China and the Belt and Road countries between 2010 and 2100 were established (Wang et al., 2017; Jiang et al., 2017b; Jiang et al., 2018a; Jiang et al., 2018b).

The Belt and Road concept is an initiative for promoting inter-regional cooperation and development through trade. Future population and economic changes in the Belt and Road countries are significant to global social and economic development, since more than $60 \%$ of the world's population and nearly half of the world's GDP are involved (WB, 2017). However, most countries in the Belt and Road region are developing nations characterized by rapid industrialization and fragile environment, and therefore, global warming poses great challenges to their continued growth (Rezai et al., 2011; Cogliano, 2016). Consequently, in this study, the future population levels, urbanization projections and GDP in each of the Belt and Road countries were analysed based on projected socioeconomic scenarios with the expectation of supporting forthcoming studies on climate change impacts and risk assessments.

\section{Study area}

A total of 65 countries, including 44 Asian countries, 20 European countries and one African country, located along the Silk Road Economic Belt and the 21st century Maritime Silk Road were included in the current study (Figure 1). The total GDP of all 65 countries throughout the Belt and Road region in 2016 was 56.5 trillion US dollars (USD) (the price in 2016, the same below), which was $47.0 \%$ of the total global GDP. Almost $62.3 \%$ (4.63 billion people) of the world's population is concentrated in this region, and the urban population of these countries accounts for $48.3 \%$ of the total (World Bank, 2017).

\section{Materials and methods}

\subsection{Data}

Various indicators, including the urban population, extent of urban land, or adjustments to the industrial structure, can be used to represents changes in urbanization. In this paper, the proportion of the urban population in the total population is used to represent the level of 


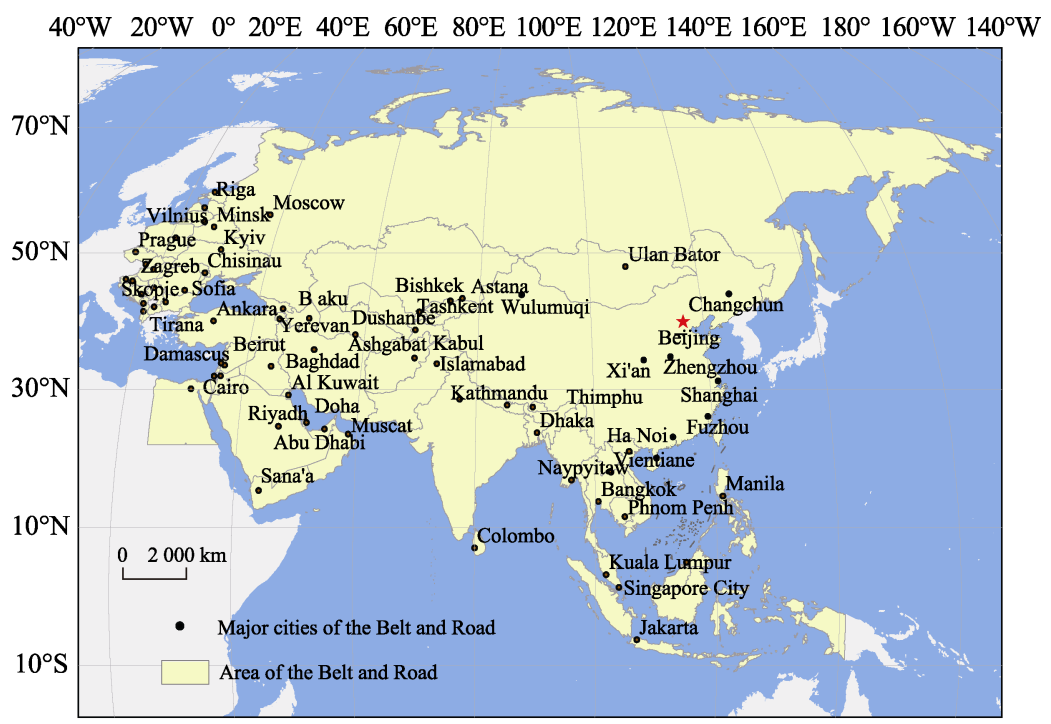

Figure 1 Location of the Belt and Road region

urbanization. The level of economic development level for the Belt and Road countries was quantified by their GDP. Furthermore, the purchasing power parity (PPP) was used to transform all prices into international USD and reflect the differences in the living and consumption levels among the various countries. The demographic and economic data of China during the historical period were derived from previous demographic and economic census and annual statistical yearbooks (NBSC, 2010). These data include the total population, urban population, total GDP, urban-rural fertility, mortality, migration, and education levels. Data from other countries in the Belt and Road region during 1990-2016 were obtained from the World Bank and the United Nations (WB, 2017; UN, 2011).

\subsection{Population and urbanization projection model}

We established projection models and parameterized them for China's population (both urban and rural) and economy. The same models were applied to all other countries in the Belt and Road region using the parameters in previous studies (Kc and Lutz, 2017; Leimbach et al., 2017).

The Population-Development-Environment (PDE) model was used to produce population projections for both urban and rural areas (Rogers, 1976). Fertility, mortality and migration rates can be defined separately for different levels of education, and transitions between different levels of education can be defined by age and sex (Lutz et al., 2001). The PDE model has been previously applied in North Africa, India, the Arabian Peninsula and Southeast Asia (Goujon, 2002; Goujon et al., 2003; Goujon et al., 2006; Goujon et al., 2007).

The initial demographic data in China for the projection, including information on fertility, mortality, migration by age, sex, and levels of education for both urban and rural populations, are based on the 6th National Population Census in 2010. For both rural and urban populations, the fertility rate, mortality rate, migration population and education level parameters under each SSP were set separately. Meanwhile, four educational categories, namely, having no education and primary, secondary and tertiary education, were used. Transitions between 
neighbouring levels by age and sex result in a shift in the population between categories. The population growth, which is defined separately for each educational category, is represented by different fertility, mortality and migration rates in the model.

The assumptions for the abovementioned factors in each SSP scenario are shown in Table 1 (Kc and Lutz, 2017). "Medium" means maintaining the current development trend. "High/Low" means that the future trend is higher/lower than the current status. For SSP1, low fertility and low mortality due to incremental investments in education levels and health might lead to a relatively small population size. For SSP2, all parameters are assumed to be medium. For SSP3, progress towards development goals is assumed to be weakened by focusing on regional energy and food security, leading to a pathway characterized by a high fertility, a high mortality and a low education focus. For SSP4, numerous countries are at low levels of development, and continued levels of low fertility, medium mortality and medium migration are assumed in addition to low education. SSP5 stresses technological progress, and thus, a low fertility, a low mortality and a high education are assumed; additionally, the level of migration is assumed to be high due to globalization and the availability of highly marketable skills.

Table 1 Assumptions of the key influencing factors on the population and economy for the SSPs

\begin{tabular}{llllll}
\hline & SSP1 & \multicolumn{1}{c}{ SSP2 } & SSP3 & SSP4 & SSP5 \\
\hline Fertility & Low & Medium & High & Low & Low \\
Mortality & Low & Medium & High & Medium & Low \\
Migration & Medium & Medium & Low & Medium & High \\
Education & High $(F T)$ & Medium $(G E T)$ & Low (CER) & Low (CER) & High (FT) \\
TFP growth & Medium & Medium & Low & Medium & High \\
\hline
\end{tabular}

(FT: fast track, GET: global education trend, CER: constant enrolment rate, TFP: total factor productivity)

For all countries throughout the Belt and Road region except China, the values of specific parameters are established in reference to previous studies by the International Institute for Applied Systems Analysis (IIASA) (Kc et al., 2013). Because the current population parameters adopted by IIASA differ from China's actual national conditions to some extent, we reset the population parameters of China under different assumptions. The initial population and specific projection parameter assumptions in the SSPs regarding the education levels and fertility, mortality, and migration rates in China are presented below.

\subsubsection{Fertility}

The recorded total fertility rate (TFR) in 2010 was 1.18 (0.97 and 1.44 for the urban and rural population, respectively), much less than the TFR estimated by various domestic and international demographers, which was approximately 1.45 (Zhao et al., 2010; Basten et al., 2013; Zeng et al., 2014). Recalculated urban and rural fertility rates in current study were 1.19 and 1.77 in 2010 , respectively.

Before 2014, the strong influence of the one-child policy on population growth resulted in a lower fertility rate throughout China (Feng et al., 2012; Cameron et al., 2013). After a universal two-child policy implemented in 2016, baby boom is expected to take place. The impact of this birth policy is expected to be much larger in urban areas than in rural areas because current fertility level in the latter is higher than that in the former, and most rural 
couples have already been allowed to foster two children (Wang, 2015). Therefore, urban and rural fertility growth rates were estimated to reach $35 \%$ and $7 \%$ compared to 2010 , respectively, after the implementation of the universal two-child policy (Zeng et al., 2014; Zeng et al., 2016).

Differences among the low, medium, and high fertility assumptions were based on research from the Vienna Institute of Demography (VID) (Basten et al., 2013; Goujon et al., 2013). In the high fertility assumption, the TFR is $20 \%$ higher than the medium fertility assumption by 2030 and $25 \%$ higher by 2050 , while the low fertility assumption is $20 \%$ lower than the medium fertility assumption by 2030 and 25\% lower by 2050 (Kc and Lutz, 2017). The low, medium and high levels of fertility in China are shown in Table 2.

Table 2 Assumptions of total fertility rate for the urban and rural populations in China

\begin{tabular}{cllllll}
\hline Year & & 2010 & 2020 & 2030 & 2040 & 2050 \\
\hline \multirow{3}{*}{ Urban } & Low & 1.19 & 1.24 & 1.29 & 1.25 & 1.21 \\
& Medium & 1.19 & 1.61 & 1.61 & 1.61 & 1.61 \\
& High & 1.19 & 1.56 & 1.93 & 1.97 & 2.01 \\
& Low & 1.19 & 1.35 & 1.51 & 1.46 & 1.42 \\
& Medium & 1.64 & 1.89 & 1.89 & 1.89 & 1.89 \\
& High & 1.19 & 1.61 & 1.61 & 1.61 & 1.61 \\
& Low & 1.19 & 1.28 & 1.37 & 1.31 & 1.25 \\
& Medium & 1.45 & 1.72 & 1.71 & 1.68 & 1.66 \\
& High & 1.19 & 1.61 & 1.61 & 1.61 & 1.61 \\
\hline
\end{tabular}

\subsubsection{Mortality}

The life expectancies were calculated by examining mortality at all ages. According to census data, the life expectancies of urban inhabitants were 77.9 and 72.4 for females and males, respectively, while those of rural inhabitants were 73.9 and 70.1 for females and males.

For the medium assumption scenario, life expectancies are assumed to approach those of developed countries with a constant increase of two years per decade. For the low (high) mortality assumption, the life expectancies are assumed to increase by one year shorter (longer) than the medium scenario. Moreover, a higher level of education corresponds to a longer life expectancy, and thus, a five-year difference was assumed between population with no education and tertiary education. The difference between the population with no education and primary education is assumed to be one year, and a two-year difference is assumed for the populations with secondary and tertiary education levels (Kc and Lutz, 2017).

\subsubsection{Migration}

The initial migration population in 2010 was calculated by census data. Most studies suggest that the migration rates for inter-provincial and urban-rural areas are slowing down due to economic growth and the narrowing of the regional development gap (Sun et al., 2011; Bell et al., 2015; Zhu et al., 2016). For the medium assumption scenario, the net migration decreases by $20 \%$ per 5 years before 2030 , and the scale of migration will continue to decline afterwards. By the end of this century, the migration rates between the urban and rural populations will gradually decrease to 0 . For the low (high) migration assumption, the mi- 
gration rate is assumed to be $50 \%$ lower (higher) than the medium scenario.

\subsubsection{Education}

In 2010, the enrolment ratios for primary, secondary (junior and senior high school) and tertiary (graduate and above) schools in China were $0.96,0.93$ and 0.27 , respectively, based on census data. The enrolment rates for primary, secondary and tertiary education in South Korea were $1.00,1.00$ and 0.93 , respectively, thereby representing high levels of enrolment. For the FT scenario, the transitions are assumed to reach high rates of enrolment by 2050 and thereafter. For the GET scenario, the transitions are assumed to reach medium rates of enrolment with values reaching $0.98,0.96$ and 0.60 by 2050 , and then, will remain unchanged. For the CER scenario, the transitions will remain at the current level.

\subsection{Economic model and parameters}

The Cobb-Douglas model was used to produce GDP projections, and the total factor productivity, capital stock and labor input were considered as the most important factors of economic projection. The GDP in year $t$ can be calculated by:

$$
G(t)=K(t)^{a} \cdot L(t)^{1-a} \cdot T F P
$$

where $G$ denotes the GDP, $K$ denotes the capital stock, $\alpha$ is the output elasticity on capital, $L$ is the labour input, and TFP is total factor productivity with 2010 as the initial year, $K$ was calculated by the following equation (Leimbach et al., 2017):

$$
K(t)=\left[\frac{K(t-1)}{L(t-1)}\right]^{(1-\alpha(t-1)) /(1-\alpha(t))} \cdot\left[\frac{\alpha(t) \cdot G(t-1)}{P_{k} \cdot K(t-1)} \cdot \frac{T F P(t)}{T F P(t-1)}\right]^{(1 /(1-\alpha(t)))} \cdot L(t)
$$

where $P_{k}$ denotes the price of capital and was assumed to 0.12 for all SSPs. GDP, TFP, K, L, and $\alpha$ in the historical period were derived from the statistical yearbook of China.

$L$ is a function of the effective factor on labour and the actual number of workers, it can be calculated by:

$$
L=\sum_{g} E \cdot L F P(g) \cdot P(g)
$$

where $g$ represents different age groups: 15-64 years and 65+years, $E$ is educational level, LFP is the labor force participation, and $P$ is the working age population. $E$ and $P$ were derived from the SSP population projections in this paper. According to the data of the 6th National Population Census, the LFP rate in 2010 was 0.77 for the population aged 15-64 years and 0.19 for the population aged $65+$ year, respectively. The assumptions of future LFP were based on studies from the Potsdam Institute for Climate Impact Research (PIK), and assumed to reach $0.7,0.7,0.6,0.77$ and 0.8 for the population aged $15-64$ years under SSP1-5 respectively, and 0.15 for the population aged $65+$ years under all SSPs.

TFP is the ratio of output to total factor input and was estimated by Solow model (Hawksworth, 2006). $\alpha$ was assumed to a time-variant elastic coefficient of output with respect to capital (Kaldor, 1961). Future assumptions of TFP and $\alpha$ were referred to the result of Leimbach et al. (2017). The long-term TFP growth rates were assumed to $0.7 \%, 0.7 \%$, $0.35 \%, 0.7 \%$ and $1.05 \%$ per year for SSP1-5 respectively, and $\alpha$ will reach $1.9-3.75$ for SSP1-5. 


\section{Results}

\subsection{Socioeconomic status in the Belt and Road region}

Countries throughout the Belt and Road region exhibit dense population and thriving economy, and the overall socioeconomic level has developed rapidly over the past 30 years. The trends of the population size, urbanization level and GDP in the Belt and Road region are shown in Figure 2. From 1990 to 2016, the population continued to grow from 3.4 to 4.6 billion, increasing by $35 \%$. The main change during this period is reflected in the growth of the urban population, which doubled from 1.1 billion in 1990 to 2.2 billion in 2016. A rapid rise in the urbanization rate from $33.3 \%$ to $48.3 \%$ can also be observed. In contrast, the rural population basically remained unchanged. The total GDP showed a surprising growth trend in the Belt and Road region, especially after 2000. The GDP increased 6.8 times from 1990 to 2016, reaching 56.5 trillion. It is worth mentioning that China played an important role in the overall GDP growth of this region with a substantial increase in its GDP from 1.1 to 21.4 trillion.

The population size, urbanization level and GDP of each country throughout the Belt and Road region in 2016 are shown in Figure 3. The population in China reached 1.38 billion, ranking as the most populated nation among the Belt and Road countries. The other 43 countries in Asia had a total population of 2.84 billion. Among these nations, five countries had populations exceeding 0.1 billion. Most of the 20 European countries are located in Central and East Europe with a total population of only 0.3 billion. Furthermore, most of the countries along the 21 st Century Maritime Silk Road are densely populated. This is espe-

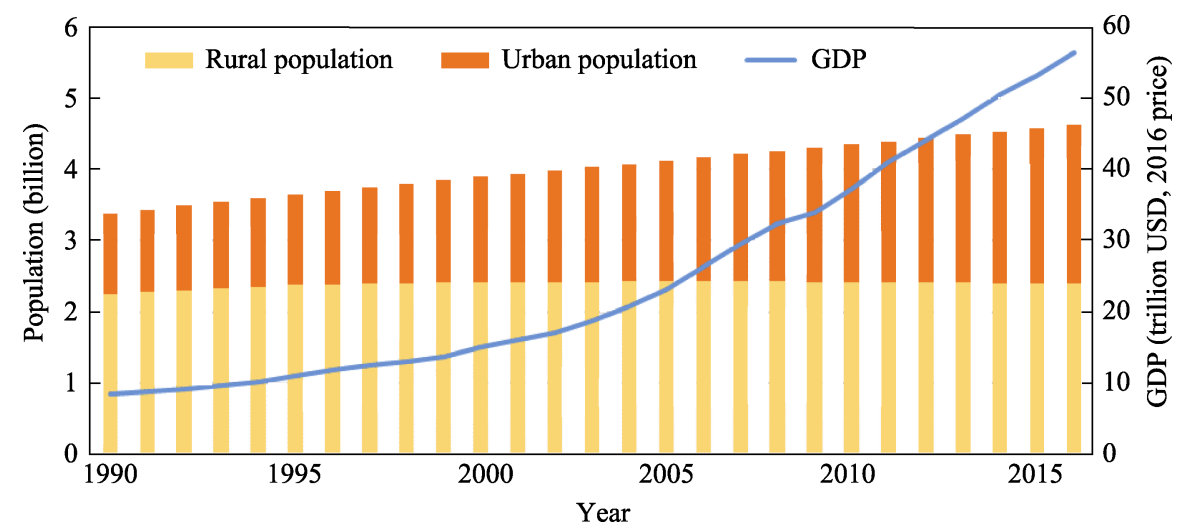

Figure 2 Changes in the population, urbanization level and GDP in the Belt and Road region for 1990-2016
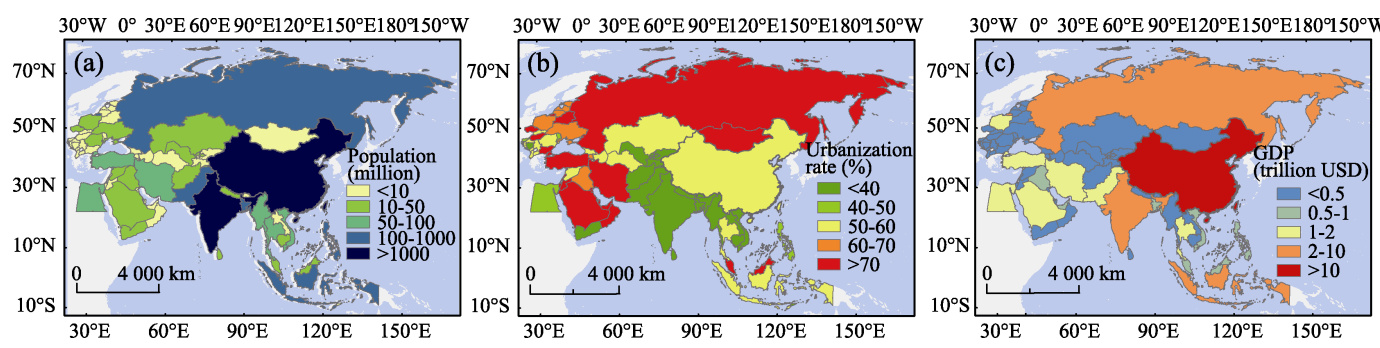

Figure 3 Distribution of the population (a), urbanization (b) and economy (c) in the Belt and Road countries in 2016 
cially true for the countries in South and Southeast Asia, this region has the highest population density in the world with an average of more than 270 people per square kilometre. In contrast, the countries along the Silk Road Economic Belt have a low population density with an average of only 26 people per square kilometre.

Nearly $50 \%$ of the population in the Belt and Road countries resides in urbanized areas, and there are significant differences in the geographical distribution of the population among the different regions. In general, Central Europe and East Europe have higher urbanization levels. Most of the countries therein have urbanization rates exceeding $60 \%$; Bulgaria is the most urbanized at 74.3\%. Meanwhile, the countries in East Europe and Central Asia have vast territories with sparse populations; nevertheless, over $50 \%$ of the population in this region is concentrated in urban areas. Most countries in the Middle East rely on oil output to obtain capital, and they have a very high urbanization level (> 70\%). The combined urban population of Saudi Arabia and Kuwait is $>90 \%$. In South Asia and Southeast Asia, where the population is dense and the economy is relatively less developed, the level of urbanization is usually low; less than half of the developing countries have urban populations of about $50 \%$, and the number is less than $20 \%$ in both Nepal and Sri Lanka.

As of 2016, the total GDP in the Belt and Road region reached 56.5 trillion USD, accounting for $47 \%$ of the total global economy. Countries located in Central Europe had a relatively large GDP per capita, but the total economy was relatively small (2.8 trillion). In contrast, the total GDP of the countries in East Europe and Central Asia was 5.2 billion USD. Russia boasted a GDP of 3.4 trillion USD, while the other countries possessed relatively small economies with less than 500 billion USD each. The economies of the countries in the Middle East were relatively high. Over half of those countries had a national GDP exceeding 500 billion USD. Developing countries are mainly distributed in South and Southeast Asia. Although the level of industrialization among these nations is relatively low, the total economic volume is large. The total GDP of the 25 countries in South and Southeast Asia in 2016 was 39.5 trillion USD, accounting for nearly $70 \%$ of the GDP in the Belt and Road region. Meanwhile, China had the highest GDP of 21.4 trillion, accounting for $37.9 \%$ of the total GDP throughout the Belt and Road region.

\subsection{Population, urbanization and economic scenarios (2020-2050)}

Socioeconomic scenarios of the population, urbanization and economy throughout the Belt and Road region are shown in Figure 4. Under different SSPs, the populations will increase in the future. As a result of the relatively high fertility rates, population growth will be the fastest under SSP3. The population will increase by about $8 \%$ every ten years from 2020 to 2050 and reach 6.0 billion in 2050.The population trajectories under SSP 1 and SSP5 basically overlap due to similar fertility and mortality assumptions. The population will increase by $2 \%$ every ten years from 2020 to 2050 and reach about 5.0 billion in 2050 . By the mid-21st century (2050), visible differences are projected with the largest difference of 1.3 billion between SSP3 (highest) and SSP5 (lowest).

With continuing globalization, both the urbanization and economic levels throughout the Belt and Road region will continue to increase. The proportion of the urban population to the total population will continue to grow at a rate of $1.4 \%-7.5 \% / 10 \mathrm{a}$. It will grow rapidly under SSP1, SSP 4 and SSP5, and reach $75 \%$ by 2050 under all three pathways. The urbanization 
growth rate for SSP2 is moderate with an urbanization level reaching $63 \%$ in 2050 . In contrast, the low level of economic development for SSP3 will lead to a relatively slow urbanization of approximately $49 \%$ in 2050 .

During 2020-2050, the economy throughout the Belt and Road region will develop greatly with the GDP increasing by 2.1, 1.7, 1.1, 1.5 and 3.8 times under SSP1, SSP2, SSP3, SSP4, and SSP5, respectively, approximately $29 \%-56 \%$ every ten years. The economy will maintain its momentum of rapid development under SSP5 with the total GDP increasing 179 trillion and reaching 243 trillion USD by 2050, which is over double greater than that under the slowest development pathway (SSP3). SSP2 represents the pathway that will maintain the current development trend, in which the GDP will increase 108 trillion and reach 172 trillion USD by 2050. Meanwhile, SSP1 will maintain a rapid growth rate, and the GDP will increase 135 trillion and reach 200 trillion USD. For SSP4, countries will show an unbalanced economic development with the GDP more concentrated in a few countries, and the total GDP will reach approximately 160 trillion USD by 2050 . The economy will be the poorest under SSP3, but it will show a continuously rising trend and reach a maximum of 134 trillion USD in 2050.
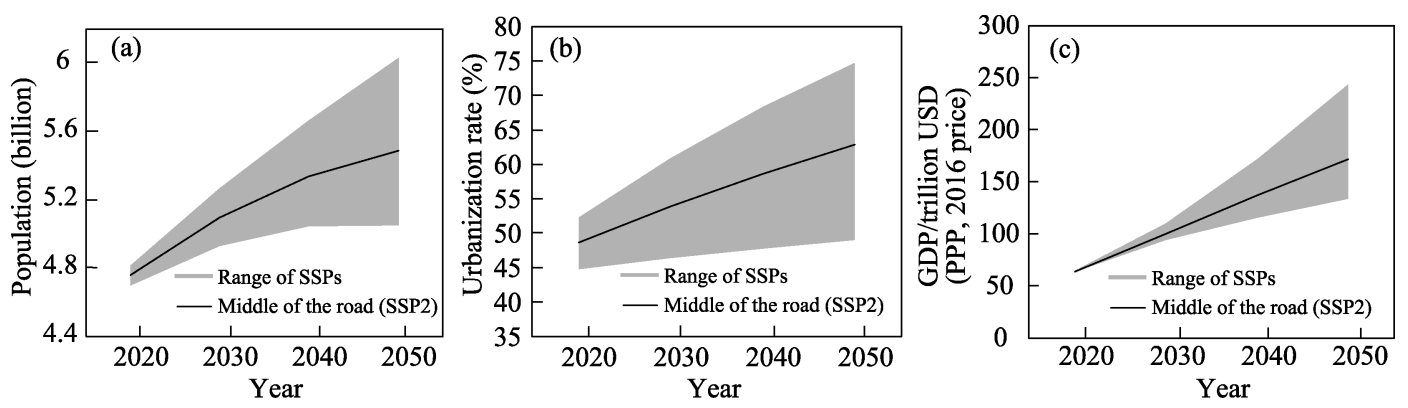

Figure 4 Projections of the population (a), urbanization (b) and economy (c) in the Belt and Road region for $2020-2050$

\subsection{Spatial variations of socioeconomy in 2050 with relative to 2016}

Figure 5 shows the spatial variations of the annual population, urbanization and GDP throughout the Belt and Road region in 2050 with relative to 2016 . Because of the low fertility, most countries in Central and Eastern Europe, as well as Russia, have a declining population in all pathways. Moldova, which will have the most severe population decline, especially in the SSP1, SSP2 and SSP5 pathways, will reduce its population by about $40 \%$ in 2050 compared to 2016. Other countries in these regions, such as Lebanon, Tajikistan, and Georgia, will also reduce by more than $20 \%$ under certain pathways. These results were shown in the Table 3. In contrast, the population of countries in the Middle East, south Asia and Central Asia are expected to increase considerably under all three pathways, and the populations in Bahrain, Yemen, Israel, Afghanistan and United Arab Emirates will even double. This feature is determined not only by the fertility rate of each country, but also the medium and high levels of mobility under these three pathways, which will bring a large number of labour force to countries with relatively high economic levels, such as Israel and United Arab Emirates. As SSP3 and SSP4 adopt a rapid population development scenario, the populations of most countries, especially those in Central Asia and the Middle East, will 
increase with relative to 2016 by above $75 \%$. Under these two pathways, countries are unevenly developed and lack communication. The populations of countries with high fertility rates will increase further. Afghanistan and Yemen will be the two countries with the fastest population growth, with more than $150 \%$ growth in 2050 compared to 2016.

Compared with 2016, the proportion of the urban population in each country will change greatly. Urbanization will develop rapidly in South Asia, West Asia and Central Asia. These regions are the areas with low urbanization level at present. In the future, with the improvement of the overall economic level, the urban population accounts for more than $80 \%$ of the total population in most countries under SSP1, SSP4 and SSP5. Nepal, Cambodia and Sri Lanka will be the three countries with the fastest growing urbanization rate, and the proportion of urban population to total population will increase from about $20 \%$ to over $50 \%$, with a growth rate of more than $160 \%$. Under the medium pathway (SSP2), the development speed of urbanization is in the middle level. The fastest growing urbanization rate in the three countries of Nepal, Cambodia and Tajikistan will increase by $103 \%, 87 \%$ and $82 \%$ respectively. Under the above four pathways, the proportion of urban population to the total population will increase in all countries. The countries with the slowest growth rate have generally reached a high level of urbanization very early. Under the slowest urbanization pathway (SSP3), in most countries, the growth rate of urbanization will be less than $10 \%$, except for some countries in Central Asia and South Asia. The fastest growing countries will be Nepal (46\%), Bosnia-Herzegovina (41\%) and Cambodia (33\%). In Southeast Asia, such as Thailand and Indonesia, as well as Mongolia, the urbanization rate will be reduced by about $21 \%, 9 \%$ and $7 \%$ respectively.

With relative to the 2016 level, the GDP in most countries will be more than double in 2050 under SSP1 and SSP2. Due to the sufficient labour, South Asia, Southeast Asia and West Asia will experience the fastest economic development with their GDP generally triple in 2050, and the GDP of Timor-Leste and Syria is expected to increase tenfold. Under SSP3 and SSP4, the GDP will grow slowly, especially in Europe, Central Asia and Southeast Asia. Some countries, such as Azerbaijani and Myanmar, will even show a negative economic growth of $-11 \%$ and $-28 \%$, respectively. Countries with faster growth will be in South Asia and West Asia, and increase over 8 time in Syria (1235\%), Timor-Leste (977\%) and Bhutan (845\%). Under SSP5, the GDP in almost all countries will double the 2016 level, and economic growth will be relatively slow only in a few eastern European countries. The three countries with the fastest economic growth (Syria, Timor-Leste and Bhutan) will experience an increase of 18, 16 and 13 times, respectively, and the three countries with the slowest growth will also experience an increase of $63 \%, 99 \%$ and $102 \%$, respectively. In summary, South Asia and West Asia will experience rapid growth compared with 2016, while the GDP growth will be relatively slow in Central Europe and East Europe under most of the pathways.

\subsection{Spatial patterns in 2050}

Figure 6 compares the percentages of countries in the Belt and Road region that will reach certain levels of the annual population, urbanization and GDP in 2050. The populations in the various countries under the different pathways will be similar, but the levels of the economy 


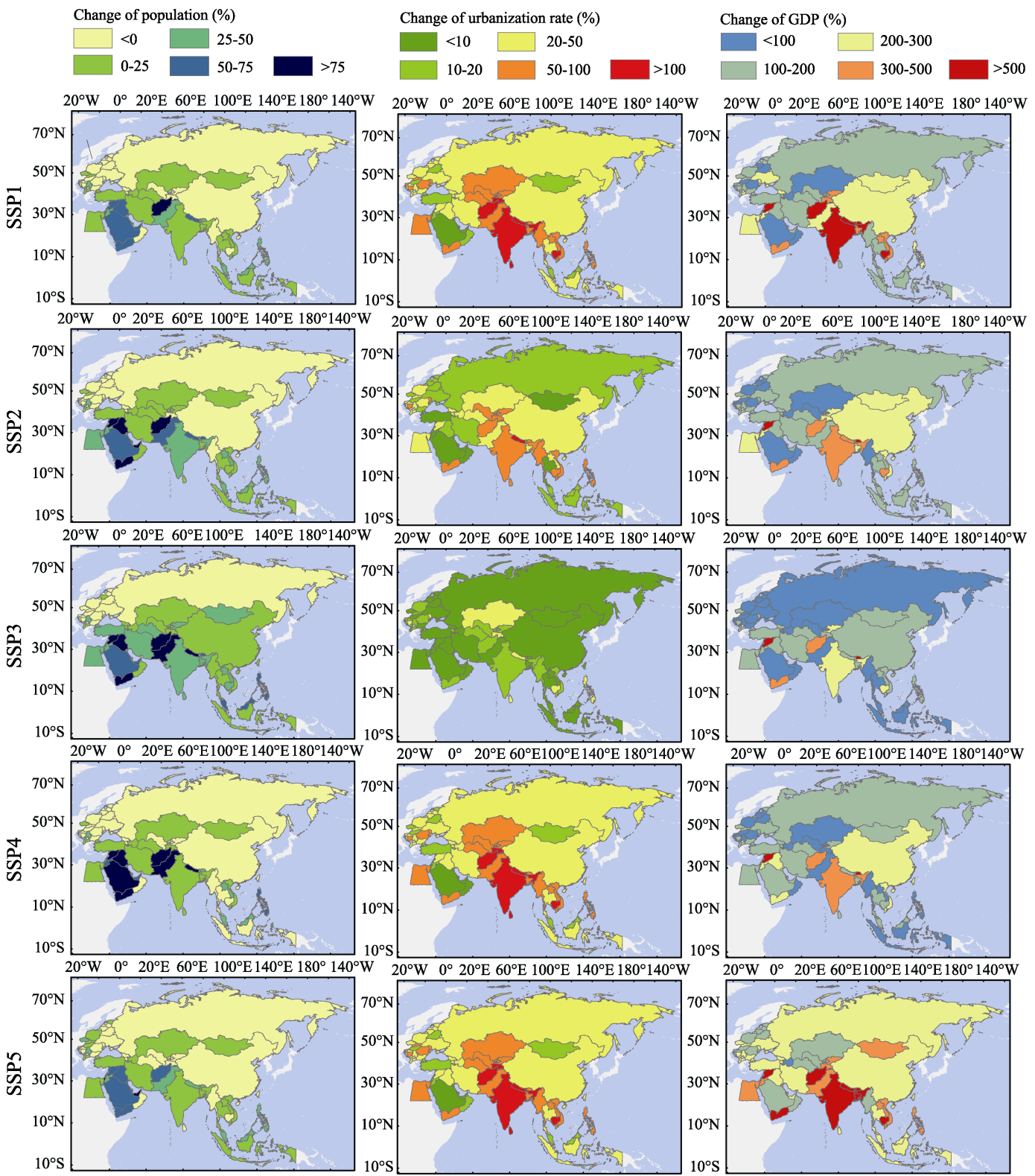

Figure 5 Changes in the population, urbanization and GDP throughout the Belt and Road region in 2050 compared with 2016

and urbanization will be significantly different under some of the pathways in 2050 .

For SSP1, the population in nearly half of the countries will reach 10 million, and India and China will be the only two countries with a population exceeding 1 billion. The urban population of almost all countries will account for over $50 \%$ of their total population, and more than $80 \%$ of the countries' urbanization rates will exceed $70 \%$. Moreover, the economic development will be relatively fast. The GDP in $31 \%$ of the countries will exceed 1 trillion USD, while the GDP of India and China will exceed 10 trillion USD, reach 69 trillion and 55 trillion respectively. 
Table 3 Three countries with the fastest and slowest growth in 2050 with relative to 2016

\begin{tabular}{|c|c|c|c|c|c|c|}
\hline \multirow{2}{*}{$\begin{array}{c}\text { Countries } \\
\text { (Rate of } \\
\text { changes) }\end{array}$} & \multicolumn{2}{|c|}{ Population } & \multicolumn{2}{|c|}{ Urbanization } & \multicolumn{2}{|c|}{ GDP } \\
\hline & Top three & Last three & Top three & Last three & Top three & Last three \\
\hline \multirow{3}{*}{ SSP1 } & Bahrain (108\%) & Moldova $(-40 \%)$ & Nepal (195\%) & Israel (1\%) & TLS (1594\%) & Azerbaijan $(26 \%)$ \\
\hline & Afghanistan (75\%) & Lebanon $(-22 \%)$ & Cambodia (167\%) & Kuwait (2\%) & Syria $(1505 \%)$ & Singapore $(62 \%)$ \\
\hline & $\operatorname{ARE}(72 \%)$ & Tajikistan $(-19)$ & Sri Lanka (160\%) & Lebanon (5\%) & Bhutan $(949 \%)$ & Kazakhstan (67\%) \\
\hline \multirow{3}{*}{ SSP2 } & Bahrain (125\%) & Moldova $(-39 \%)$ & Nepal $(103 \%)$ & Israel (1\%) & Syria $(1235 \%)$ & Azerbaijan (7\%) \\
\hline & Afghanistan (117\%) & Lebanon $(-17 \%)$ & Cambodia (87\%) & Kuwait (2\%) & TLS (977\%) & Myanmar (40\%) \\
\hline & Yemen $(105 \%)$ & Belarus $(-14)$ & Tajikistan $(82 \%)$ & Lebanon (5\%) & Bhutan $(845 \%)$ & Kazakhstan (47\%) \\
\hline \multirow{3}{*}{ SSP3 } & Afghanistan (164\%) & Moldova $(-24 \%)$ & Nepal (46\%) & Thailand $(-21 \%)$ & Syria $(1235 \%)$ & Azerbaijan $(-11 \%)$ \\
\hline & Yemen $(152 \%)$ & Hungary $(-22 \%)$ & $\mathrm{BIH}(41 \%)$ & Indonesia $(-9 \%)$ & TLS (977\%) & Myanmar (3\%) \\
\hline & TLS (143\%) & Poland $(-18 \%)$ & Cambodia (33\%) & Mongolia (-7\%) & Bhutan $(845 \%)$ & Romania (18\%) \\
\hline \multirow{3}{*}{ SSP4 } & Afghanistan (153\%) & Moldova $(-42 \%)$ & Nepal (195\%) & Israel (1\%) & Syria $(857 \%)$ & Myanmar (-28\%) \\
\hline & Yemen (149\%) & Lebanon $(-$ & Cambodia (167\%) & Kuwait (2\%) & Bhutan $(670 \%)$ & Azerbaijan (7\%) \\
\hline & Iraq $(127 \%)$ & Belarus $(-20 \%)$ & Sri Lanka (160\%) & Lebanon $(5 \%)$ & TLS (442\%) & Romania (47\%) \\
\hline \multirow{3}{*}{ SSP5 } & Bahrain (144\%) & Moldova $(-50 \%)$ & Nepal (195\%) & Israel (1\%) & Syria $(1888 \%)$ & Azerbaijan (63\%) \\
\hline & Israel $(103 \%)$ & Tajikistan $(-38 \%)$ & Cambodia (167\%) & Kuwait (2\%) & TLS (1621\%) & Singapore $(99 \%)$ \\
\hline & ARE $(101 \%)$ & Georgia $(-27 \%)$ & Sri Lanka $(160 \%)$ & Lebanon ( $5 \%)$ & Bhutan $(1265 \%)$ & Lithuania (102\%) \\
\hline
\end{tabular}

ARE: United Arab Emirates, TLS: Timor-Leste, BIH: Bosnia and Herzegovina
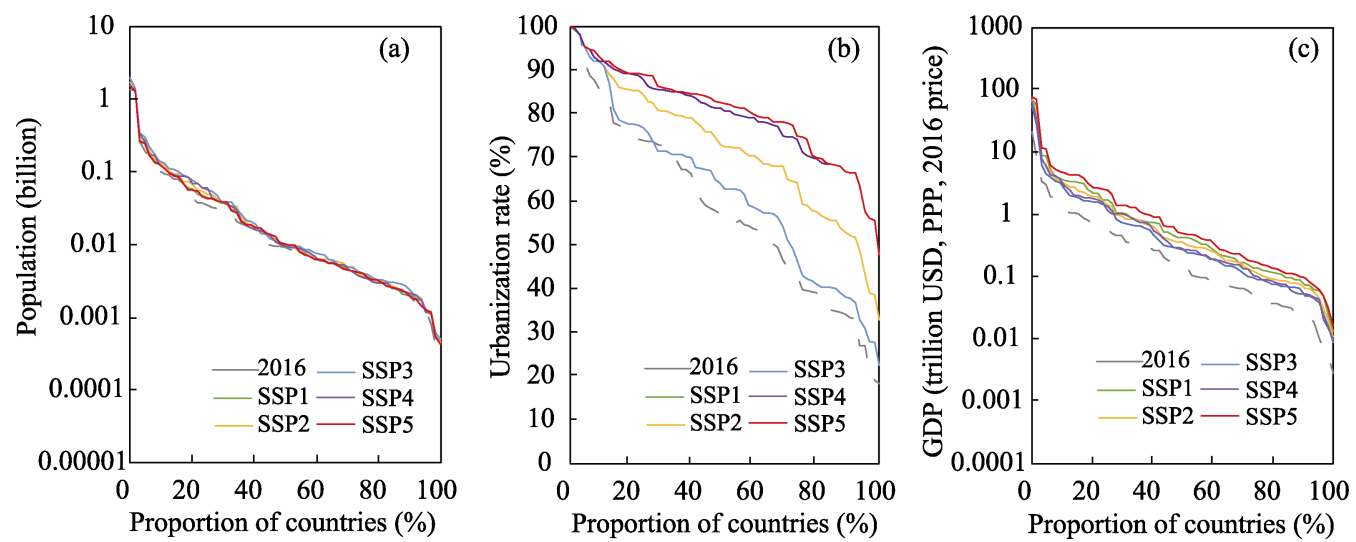

Figure 6 Comparisons of the annual population (a), urbanization (b) and GDP (c) in the Belt and Road region in 2050 with the 2016 levels

For SSP2, the current development trend will be maintained. The demographic and economic development levels are projected to be similar to those for SSP1 in 2050, but the urbanization level will be lower. The urban population in all countries will exceed $30 \%$, and in half of the countries throughout will reach $70 \%$.

For SSP3, the populations in half of the Belt and Road countries will exceed 10 million, and in the most populous country (India) will exceed 1.9 billion. Urbanization will develop slowly with an urban population of more than $70 \%$ in only $40 \%$ of the countries. The urban population in Sri Lanka will be the lowest with urbanization level of about $23 \%$. The urban population will exceed $90 \%$ in 8 countries. Economic development will also proceed slowly. 
The GDP of only $27 \%$ of the countries will be more than 1 trillion USD. Amongst, the GDP in China and India will far exceed 10 trillion with maxima of 54.5 trillion and 28.2 trillion, respectively.

Similar to SSP1, the population growth will be relatively low for SSP4, but urbanization will rapidly develop. The GDP in $30 \%$ of the countries will exceed 1 trillion USD.

Under SSP5, the population distribution will similar to SSP1. The urbanization and economic levels will develop rapidly. The GDP in approximately 38\% of countries will exceed 1 trillion USD, and in 4 countries, including China, India, Russian and Indonesia, will exceed 10 trillion USD. China and India are expected to have a GDP exceeding 50 trillion USD and reach 77 trillion and 69 trillion USD, respectively.

\section{Conclusions and discussion}

\subsection{Conclusions}

Since The Belt and Road Initiative was proposed, it has attracted international attention. There are substantial socioeconomic differences between the Belt and Road countries, and thus, the selection of climate scenarios will significantly influence their future social and economic development. We therefore evaluate the spatial distribution and evolution of the projected population, urbanization and economic levels for 2020-2050 for the Belt and Road countries using five SSPs from the IPCC, namely, sustainability (SSP1), middle of the road (SSP2), regional rivalry (SSP3), inequality (SSP4) and fossil-fuelled development (SSP5). The following conclusions can be reached.

(1) The urban population in the Belt and Road countries nearly double from 1.1 billion to 2.2 billion during 1990-2016, but almost no change in their rural population. This led to a $15 \%$ increase in the level of urbanization. Simultaneously, the total GDP increased by 5.8 times from 8 trillion to 57 trillion USD. In 2016, the urban population accounts for $50 \%$ of the total population of the Belt and Road region. The countries along the 21 st century Maritime Silk Road are densely populated; yet, their levels of economic and urban development were relatively low. However, countries along the Silk Road Economic Belt exhibited opposite characteristics.

(2) From 2020 to 2050, the levels of urbanization and economic development will increase greatly in the Belt and Road region under the various pathways. The average rate of urbanization will increase by $1.4 \%-7.5 \% / 10 \mathrm{a}$, and reach $49 \%-75 \%$ under the five pathways. The GDP will increase by approximately $17 \%-34 \%$ every ten years. By 2050 , the total GDP in the Belt and Road region will reach 134-243 trillion USD. The growth of the total population will be relatively slow, with an average of only $2 \%-8 \%$ per decade, and reach $5.0-6.0$ billion in 2050 .

(3) Under the sustainable SSP1 and economic development-oriented SSP5 pathways, the urbanization and economy will develop rapidly but with less population growth. Under SSP2, social and economic development indicators will maintain their current trends at a moderate level. Under the unbalanced pathway of SSP4, the population and economy will grow slowly, but urbanization will develop rapidly. Under SSP3 characterized by regional competition, the population growth will be rapid, but the economic development and ur- 
banization will be relatively slow.

(4) With relative to 2016, the populations will increase in most countries in 2050, and especially obvious in Central Asia and the Middle East countries. The growth will more than double in Bahrain, Afghanistan and Yemen. The economy will develop rapidly in South Asia, Southeast Asia and West Asia, and increase by more than 10 times in Timor-Leste, Syria and Bhutan. However, the economy will grow relatively slowly in Central Europe and East Europe under most of the pathways. The trend of urbanization will be similar to that of the economy. The urbanization in South Asia, West Asia and Central Asia will develop rapidly and increase by more than $150 \%$ in Nepal, Cambodia and Sri Lanka.

\subsection{Discussion}

In consideration of policy interventions and new parameters from the latest 2010 population census, this study first projects the population, urbanization and economic levels in China and then investigates the situation in the Belt and Road region under five different SSPs. This is the first time that newly implemented policy and census data are used in socioeconomic projections extending to 2050. Accordingly, updated information and credible population scenarios allow us to produce projections that are more realistic.

The accuracy of the population projection is the basis of further economic and urbanization projections. For different countries, future fertility rates constitute the key factor in reliably projecting the population growth. Due to various uncertain determinants (e.g., urbanization and education) of the fertility rate, the populations projected using simple assumptions of the total fertility or using relatively complex total fertility rates obtained by a combination of urban and rural fertilities might overestimate the population (UN, 2011; Zeng and Wang, 2014; Zeng and Hesketh, 2016). Consequently, related sensitivity studies need to be conducted in the future.

The Belt and Road region will exhibit a varying socioeconomic status in the future, and as a result, the selection of different SSPs will likely bring about completely different results. Different SSPs provide a framework for developing new socioeconomic scenarios. They can be used for not only global climate change studies but also assessments of the development of broader sustainability. This article does not present a optimistic option for future development, but rather, simply represent future possibilities and provide appropriate references for decision makers once the situation changes. The setting of five different pathways is conducive to reduce the uncertainty in socioeconomic projection. It is obviously better than the projection with single scenario.

The SSP-based socioeconomic projections are closely related to climate change. In the upcoming IPCC sixth assessment report (AR6), SSPs will be combined with RCPs to develop new scenarios for comprehensively assessing climate change and human activities. Many forthcoming studies are expected to associate the socioeconomic scenarios in the Belt and Road region with risk assessments of climate and weather disasters.

\section{References}

Arnell N W, Lloyd-Hughes B, 2014. The global-scale impacts of climate change on water resources and flooding under new climate and socio-economic scenarios. Climatic Change, 122(1/2): 127-140. 
Basten S, Sobotka T, Zeman K, 2013. Future Fertility in Low Fertility Countries. Vienna: Vienna Institute of Demography Working Papers.

Bell M, Charles-Edwards E, Ueffing $\mathrm{P}$ et al., 2015. Internal migration and development: Comparing migration intensities around the world. Population, Space and Place, 41(1): 33-58.

Cai W, Borlace S, Lengaigne M et al., 2014. Increasing frequency of extreme El Niño events due to greenhouse warming. Nature Climate Change, 4(2): 111-116.

Chen H, Zhang Y, 2012. Sensitivity experiments of impacts of large-scale urbanization in East China on East Asian winter monsoon. Chinese Science Bulletin, 58(7): 809-815.

Cogliano J F, 2016. Climate change and international trade. Eastern Economic Journal, 42(1): 159-161.

Cuaresma J C, 2017. Income projections for climate change research: A framework based on human capital dynamics. Global Environmental Change, 42: 226-236.

Dellink R, Chateau J, Lanzi E et al., 2017. Long-term economic growth projections in the Shared Socioeconomic Pathways. Global Environmental Change, 42: 200-214.

Donat M G, Lowry A L, Alexander L V et al., 2016. More extreme precipitation in the world's dry and wet regions. Nature Climate Change, 6(5): 508-513.

Field C B, Barros V, Stocker T F et al., 2012. Managing the Risks of Extreme Events and Disasters to Advance Climate Change Adaptation: Special Report of the Tntergovernmental Panel on Climate Change. Cambridge, United Kingdom and New York, NY, USA: Cambridge University Press.

Fujimori S, Kainuma M, Masui T et al., 2014. The effectiveness of energy service demand reduction: A scenario analysis of global climate change mitigation. Energy Policy, 75: 379-391.

Goujon A, 2002. Population and Education Prospects in the Arab Region. Cairo: The American University in Cairo Press.

Goujon A, Alkitkat H, Lutz W et al., 2007. Population and Human Capital Growth in Egypt: Projections for Governorates to 2051. Laxenburg: International Institute for Applied Systems Analysis.

Goujon A, Fuchs R, 2013. The Future Fertility of High Fertility Countries: A Model Incorporating Expert Arguments. Laxenburg: International Institute for Applied Systems Analysis.

Goujon A, McNay K, 2003. Projecting the educational composition of the population of India: Selected state-level perspectives. Applied Population and Policy, 1(1): 25-35.

Goujon A, Samir K C, 2006. Past and Future of Human Capital in Southeast Asia: From 1970 to 2030. Vienna: Vienna Institute of Demography Working Papers.

Hansen G, Stone D, 2015. Assessing the observed impact of anthropogenic climate change. Nature Climate Change, 6(5): 532-537.

Hawksworth J, 2006. How big will the major emerging market economies get and how can the OECD compete. Pricewaterhouse Coopers.

Hegre H, Buhaug H, Calvin K V et al., 2016. Forecasting civil conflict along the shared socioeconomic pathways. Environmental Research Letters, 11(5): 054002.

IPCC, 2013. Climate Change 2013: The Physical Science Basis. Contribution of Working Group I to the Fifth Assessment Report of the Intergovernmental Panel on Climate Change. Cambridge, United Kingdom and New York, USA: Cambridge University Press, pp: 1535.

Jiang L, O’Neill B C, 2017a. Global urbanization projections for the Shared Socioeconomic Pathways. Global Environmental Change, 42: 193-199.

Jiang T, Wang Y J, Yuan S J et al., 2018a. Projection of population and economy in the Belt and Road countries (2020-2060). Climate Change Research, 14(2): 155-164. (in Chinese)

Jiang T, Zhao J, Cao L G et al., 2018b. Projection of national and provincial economy under the Shared Socioeconomic Pathways in China. Climate Change Research, 14(1): 50-58. (in Chinese)

Jiang T, Zhao J, Jing C et al., 2017b. National and provincial population projected to 2100 under the Shared Socioeconomic Pathways in China. Climate Change Research, 13(2): 128-137. (in Chinese)

Jones R, Patwardan A, Cohen S et al., 2014. Foundations for Decision Making. Cambridge, United Kingdom and New York, NY, USA: Cambridge University Press, 195-228.

Kaldor N, 1961. Capital accumulation and economic growth. In: Lukz, Hague (eds). The Theory of Capital. New York: St. Martin' Press.

Kc S, Lutz W, 2014. Demographic scenarios by age, sex and education corresponding to the SSP narratives. Population and Environment, 35(3): 243-260.

Kc S, Lutz W, 2017. The human core of the shared socioeconomic pathways: Population scenarios by age, sex and level of education for all countries to 2100. Global Environmental Change, 42: 181-192.

Kc S, Potancokova M, Bauer R et al., 2013. Summary of Data, Assumptions and Methods for New Wittgenstein Centre for Demography and Global Human Capital (WIC) Population Projections by Age, Sex and Level of Education for 195 Countries to 2100. Laxenburg: International Institute for Applied Systems Analysis.

Kriegler E, Edmonds J, Hallegatte S et al., 2014. A new scenario framework for climate change research: The concept of shared climate policy assumptions. Climatic Change, 122(3): 401-414. 
Leggett J, Pepper W J, Swart R J, 1992. Emissions scenarios for the IPCC: An update. In: IPCC. Climate Change 1992: The Supplementary Report to the IPCC Scientific Assessment. Cambridge: Cambridge University Press, 69-95.

Leimbach M, Kriegler E, Roming N et al., 2017. Future growth patterns of world regions - A GDP scenario approach. Global Environmental Change, 42: 215-225.

Lutz W, Butz W, Kc S et al., 2014. Population growth: Peak probability. Science, 346(6209): 561.

Lutz W, Goujon A, 2001. The world's changing human capital stock: Multi-state population projections by educational attainment. Population and Development Review, 27(2): 323-339.

Mogollón J M, Lassaletta L, Beusen A H W et al., 2018. Assessing future reactive nitrogen inputs into global croplands based on the shared socioeconomic pathways. Environmental Research Letters, 13(4): 044008.

Moss R H, Edmonds J A, Hibbard K A et al., 2010. The next generation of scenarios for climate change research and assessment. Nature, 463(7282): 747-756.

Nakicenovic N, Kolp P, Riahi K et al., 2006. Assessment of emissions scenarios revisited. Environmental Economics and Policy Studies, 7(3): 137-173.

Nakicenovic N, Swart R, 2000. Emission Scenarios IPCC Special Report on Emission Scenarios. Cambridge, United Kingdom and New York, USA: Cambridge University Press, pp: 598.

National Bureau of Statistics of China (NBSC), 2010. China Statistical Yearbook 2010. Beijing: China Statistics Press. (in Chinese)

O’Neill B C, Kriegler E, Ebi K L et al., 2017. The roads ahead: Narratives for shared socioeconomic pathways describing world futures in the 21st century. Global Environmental Change, 42: 169-180.

O’Neill B C, Kriegler E, Riahi K et al., 2014. A new scenario framework for climate change research: the concept of shared socioeconomic pathways. Climatic Change, 122(3): 387-400.

Popp A, Calvin K, Fujimori S et al., 2017. Land-use futures in the shared socio-economic pathways. Global Environmental Change, 42: 331-345.

Rezai A, Foley D K, Taylor L, 2011. Global warming and economic externalities. Economic Theory, 49(2): 329-351.

Riahi K, van Vuuren D P, Kriegler E et al., 2017. The Shared Socioeconomic Pathways and their energy, land use, and greenhouse gas emissions implications: An overview. Global Environmental Change, 42: 153-168.

Rogers A, 1976. Introduction to Multiregional Mathematical Demography. New York: Wiley.

Rohde R A, Muller R, Jacobsen R et al., 2013. A new estimate of the average earth surface land temperature spanning 1753 to 2011. Geoinformatics \& Geostatistics: An Overview, 1(1).

Rosenzweig C, Elliott J, Deryng D et al., 2014. Assessing agricultural risks of climate change in the 21 st century in a global gridded crop model intercomparison. Proceedings of the National Academy of Sciences, 111(9): 3268-3273.

Schewe J, Heinke J, Gerten D et al., 2014. Multimodel assessment of water scarcity under climate change. Proceedings of the National Academy of Sciences, 111(9): 3245-3250.

Sun B, Tang Z Y, 2011. The trend of population in urban under the factors of inter povincial migration and rural-urban migration. Northwest Population, 1(32): 64-72. (in Chinese)

Taylor K E, Stouffer R J, Meehl G A, 2012. An overview of CMIP5 and the experiment design. Bulletin of the American Meteorological Society, 93(4): 485-498.

Trenberth K E, Fasullo J T, Shepherd T G, 2015. Attribution of climate extreme events. Nature Climate Change, 5(8): 725-730.

United Nations (UN), 2011. World Population Prospects The 2010 Revision. New York: Department of Economic and Social Affairs, Population Division.

van Vuuren D P, Edmonds J, Kainuma M et al., 2011. The representative concentration pathways: An overview. Climatic Change, 109(1): 5.

van Vuuren D P, Kok M T J, Girod B et al., 2012. Scenarios in global environmental assessments: Key characteristics and lessons for future use. Global Environmental Change, 22(4): 884-895.

Wang G Z, 2015. Rethinking the problems in the research of China's family planning policy adjustment. Chinese Journal of Population Science(2): 2-15. (in Chinese)

Wang Y J, Jing C, Cao L G et al., 2017. The population patterns over China in the $1.5^{\circ} \mathrm{C}$ and $2.0^{\circ} \mathrm{C}$ warming targets. Climate Change Research, 13(4): 327-336. (in Chinese)

Weber C L, Peters G P, 2009. Climate change policy and international trade: Policy considerations in the US. Energy Policy, 37(2): 432-440.

World Bank (WB), 2017. World Development Indicators. Washington: World Bank.

Zeng Y, Hesketh T, 2016. The effects of China's universal two-child policy. The Lancet, 388(10054): 1930-1938.

Zeng Y, Wang Z, 2014. A policy analysis on challenges and opportunities of population/household aging in China. Journal of Population Ageing, 7(4): 255-281.

Zhu Y, Lin L Y, Ke W Q, 2016. Trends in internal migration and mobility: International experiences and their implications for China. Population Research, 40(5): 50-60. (in Chinese) 